\title{
Long term neuropsychological outcome after head injury: relation to APOE genotype
}

\section{K Millar, J A R Nicoll, S Thornhill, G D Murray, G M Teasdale}

See end of article for authors' affiliations

\section{Correspondence to:} Professor J A R Nicoll mailpoint 813 , level $\mathrm{E}$, Southampton General Hospital, Southampton SO16 6YD, UK; j.nicoll@soton.ac.uk

Received 4 September 2002

In revised form

20 February 2003

Accepted

26 February 2003
Background: Existing evidence suggests that some patients who sustain a head injury suffer cognitive decline many years later, and that head injury and possession of the APOE $\epsilon 4$ allele are each risk factors for Alzheimer's disease.

Objective: To determine whether late cognitive decline after head injury is more prevalent among carriers of $A P O E \in 4$.

Methods: A database of head injured patients was used. Initial assessment was at the time of their injury, between 1968 and 1985, and outcome data at six months were available. Their ages at the time of injury ranged between 2 and 70 years. A cohort of 396 subjects was reassessed at a mean of 18 years later, with determination of APOE genotype and detailed neuropyschological testing.

Results: Judging by the Glasgow outcome scale, twice as many patients had deteriorated as improved between six months after injury and the late assessment; $22.2 \%$ of APOE $\epsilon 4$ carriers had a good late outcome compared with $30.5 \%$ of non-carriers $195 \%$ confidence interval for the difference, $-0.7 \%$ to $17.2 \% ; p=0.084)$. There were no clear differences between $\epsilon 4$ carriers and non-carriers in detailed neuropsychological assessments.

Conclusions: Although this study provides additional evidence that a late decline may occur after head injury, there was no clear relation to APOE genotype. Despite the follow up interval of 15 to 25 years, the cohort is still too young (mean age 42.1 years) to assess the risk of Alzheimer's disease.
$\mathrm{E}$ vidence is accumulating that early outcome after a head injury is influenced by polymorphism of apolipoprotein $\mathrm{E}$ (APOE gene; apolipoprotein E protein). The gene has three common alleles which are designated $\epsilon 2, \epsilon 3$, and $\epsilon 4$, and encode corresponding protein isoforms (E2, E3, and E4). In a prospective clinical study using the Glasgow outcome scale, we found a significant association between the possession of APOE $\epsilon 4$ and an unfavourable outcome at six months after head injury. ${ }^{1}$ In that study of 93 patients, $\epsilon 4$ carriers were more than twice as likely as non-carriers to have a poor outcome, defined as dead, in a vegetative state, or severely disabled. Similar associations have been reported between the possession of APOE $\epsilon 4$ and the reduced likelihood of recovery of consciousness from post-traumatic coma ${ }^{23}$ and poor outcome in survivors within the first year after injury. ${ }^{34}$ In addition to an influence on relatively short term outcome, a relation was proposed between possession of APOE $\epsilon 4$ and the long term consequences for survivors of head injury. ${ }^{5}$

The few prospective studies of outcome decades after head injury have included limited information concerning the initial features of the injury, and the assessments of cognitive function that were done were relatively unsophisticated. ${ }^{67}$ Nevertheless, such studies suggest that there may be a long term decline. For example, compared with personnel who had peripheral nerve injuries during World War II, those who sustained a head injury had worse cognitive function 30 years later. ${ }^{7}$ In a large recent study 50 years after they had sustained a closed head injury, World War II veterans showed a higher prevalence of Alzheimer's disease than controls. ${ }^{8}$ In contrast, a long term review of servicemen with focal wartime brain wounds suggested preservation of cognitive abilities. ${ }^{9}$

Information on the association between head injury and long term neurodegeneration has also come from retrospective epidemiological studies of Alzheimer's disease which have identified an association with a previous head injury. ${ }^{10-15}$ The largest of these studies found that patients with Alzheimer's disease were substantially more likely to have suffered a head injury with loss of consciousness than non-demented controls. ${ }^{15}$ However, the conclusions of these retrospective studies have been criticised on the basis that they may have been subject to recall bias, and a recent large community based prospective study found no association between a head injury and Alzheimer's disease. ${ }^{16}$

Possession of APOE $\epsilon 4$ is the major genetic risk factor for sporadic Alzheimer's disease. ${ }^{17}$ An early study investigating both head injury and APOE $\epsilon 4$ as risk factors for Alzheimer's disease suggested that the interaction may be synergistic, ${ }^{18}$ but subsequent larger studies concluded that the effects of these two risk factors are additive or less than additive. ${ }^{13-15}$

Further information on the relation between traumatic brain injury, Alzheimer's disease, and the APOE genotype has come from studies of boxers. Boxers with APOE $\epsilon 4$ have more evidence of chronic brain injury than non-carriers of $\epsilon 4,{ }^{19}$ and boxers may suffer from cognitive decline as they age (dementia pugilistica)..$^{20}$

The present study took advantage of a database of patients with severe head injuries who had been studied in Glasgow from the late 1960s to the mid 1980s. The database contains information about the patients, their injury, the initial features including severity and clinical progression, and outcome as assessed by the Glasgow outcome scale ${ }^{21}$ six months after the injury. This unique dataset, taking account of early clinical indices, has allowed the investigation of a possible association between APOE genotype and very long term outcome, using detailed neuropsychological assessments. Specifically, we aimed to address the hypothesis that long term survivors of head injury show impairment of cognitive function many years after their injury, controlling for outcome

Abbreviations: $A P O E$, gene for apoliprotein $E ; G H Q$, general health questionnaire; GOSE, extended Glasgow outcome scale; MMSE, mini-mental state examination; NART, national adult reading test; $\mathrm{SCL}$, symptom check list; WMS, Wechsler memory scale 
Table 1 Early clinical data

\begin{tabular}{|c|c|c|c|}
\hline & $\begin{array}{l}\text { Known to be } \\
\text { dead }\end{array}$ & $\begin{array}{l}\text { Contacted and } \\
\text { assessed }\end{array}$ & Remainder \\
\hline Sample size & 242 & 396 & 495 \\
\hline \multicolumn{4}{|l|}{ Year of injury } \\
\hline Median & 1979 & 1980 & 1976 \\
\hline Interquartile range & 1976 to 1983 & 1977 to 1985 & 1974 to 1983 \\
\hline \multicolumn{4}{|l|}{ Age at injury (years) } \\
\hline Mean & 46.7 & 23.9 & 27.0 \\
\hline SD & 17.4 & 15.3 & 17.6 \\
\hline Male & $194(80.2)$ & $291(73.5)$ & $397(80.2)$ \\
\hline \multicolumn{4}{|l|}{ Cause of injury } \\
\hline Road traffic accident & 92 (39.0) & $261(66.6)$ & $300(61.3)$ \\
\hline Fall under influence of alcohol & $82(34.7)$ & $28(7.1)$ & $56(11.5)$ \\
\hline Other & $62(26.3)$ & $103(26.3)$ & $133(27.2)$ \\
\hline Major extracranial complication & $51(21.2)$ & $155(39.2)$ & $173(35.0)$ \\
\hline Lucid interval & $82(36.0)$ & $69(18.1)$ & $111(23.2)$ \\
\hline \multicolumn{4}{|l|}{ GCS at $A \& E$} \\
\hline $3-8$ & $151(74.4)$ & $272(85.0)$ & $297(79.8)$ \\
\hline $9-12$ & $17(8.4)$ & $21(6.6)$ & $39(10.5)$ \\
\hline $13-15$ & $35(17.2)$ & $27(8.4)$ & $36(9.7)$ \\
\hline Non-reacting pupils at $A \& E$ & $44(22.4)$ & $39(11.8)$ & $43(11.4)$ \\
\hline \multicolumn{4}{|l|}{ Injury type * } \\
\hline Diffuse & $59(24.4)$ & $233(58.8)$ & $265(53.5)$ \\
\hline Evacuated mass & $154(63.6)$ & $140(35.4)$ & $187(37.8)$ \\
\hline Non-evacuated mass & $29(12.0)$ & $23(5.8)$ & $43(8.7)$ \\
\hline \multicolumn{4}{|l|}{ GOS at six months } \\
\hline Vegetative/severe disability & $129(55.4)$ & $101(25.6)$ & $116(23.6)$ \\
\hline Moderate disability & $57(24.5)$ & $126(31.9)$ & $151(30.7)$ \\
\hline Good recovery & $47(20.2)$ & $168(42.5)$ & $225(45.7)$ \\
\hline
\end{tabular}

at six months, and that this impairment could be more prevalent among APOE $\epsilon 4$ carriers.

\section{METHODS}

\section{Ethical approval}

The Southern General Hospital ethics committee approved the study. Additional approval came from all 14 regional health boards in Scotland which were contacted to confirm registration of patients with general practitioners. Approval was also obtained from the local general practitioner subcommittees, as we required the consent of the patient's GP before contacting the patient.

\section{Sample}

The population comprised the database of 1133 patients who had sustained a severe head injury and had been studied in the acute stage in the Department of Neurosurgery, Institute of Neurological Sciences at the Southern General Hospital, Glasgow, between 1968 and 1985. For the entire group of 1133 patients the age range at injury was $<1$ to 82 years. For the 396 patients who were assessed, the age range was 2 to 70 years. Patients had to have survived for at least six months following their original injury to be included in the study. The tracing of patients began with the general practitioner recorded at the time of discharge from hospital and progressed if necessary to searches of the community health index, SMRI forms, and registrar general data to identify those lost to follow up through death.

\section{Patient contact}

The general practitioner of the patients who were traced was contacted, informed of the details of the study, and asked for permission to contact the patient. Following GP consent, a letter was written to each patient informing them of the study and asking them to participate by completing a consent form. A contact telephone number was offered to allow the patient an opportunity to discuss the study further with the team before consenting. When the patient was unable to give consent, the next of kin or legal guardian was consulted. The initial contact letter was followed up by a telephone call, where possible, to discuss potential participation in the study.

\section{Determination of APOE genotype}

APOE genotypes were determined from buccal swabs which were obtained by rubbing a cytology brush on the inner surface of the patient's cheek for approximately 15 seconds. The brush was then inserted into an Eppendorf tube containing $400 \mu \mathrm{l}$ of ethanol and agitated. Following storage at $4^{\circ} \mathrm{C}$, samples were centrifuged at $11600 \times g$ for 30 seconds to pellet the cells and the ethanol was decanted. The pelleted samples were dried on a heat block at $55^{\circ} \mathrm{C}$ for 30 minutes, digested with proteinase $\mathrm{K}(100 \mu \mathrm{l} / \mathrm{ml})$ at $56^{\circ} \mathrm{C}$ for one hour, and then heated to $95^{\circ} \mathrm{C}$ for 10 minutes to inactivate the proteinase $\mathrm{K}$. Using 0.8 $\mu \mathrm{l}$ of the proteinase digest as the template, hot start polymerase chain reaction (PCR) amplification was undertaken with standard primers ${ }^{22}$ in a reaction volume of $15 \mu \mathrm{l}$ (40 cycles of consisting $94^{\circ} \mathrm{C}$ for one minute, $65^{\circ} \mathrm{C}$ for one minute, and $72^{\circ} \mathrm{C}$ for two minutes). The PCR products were digested with Hhal for a minimum of three hours. The products of digestion were separated according to size by polyacrylamide gel electrophoresis, stained with ethidium bromide, and viewed by ultraviolet transillumination.

\section{Assessment}

Early clinical data

Data were available for all patients concerning the nature and severity of the head injury judged by clinical features, including the Glasgow coma score, ${ }^{23}$ and pupil reactivity, treatment, and outcome at six months on the Glasgow outcome scale. ${ }^{21}$

\section{Long term outcome}

The comprehensive assessment was conducted in the research laboratory, or, where this proved impracticable, in the patient's home. 
Table 2 Early clinical data according to APOE genotype assessment

\begin{tabular}{|c|c|c|}
\hline & \multicolumn{2}{|l|}{ APOE genotype } \\
\hline & $\epsilon 4$ negative & $\epsilon 4$ positive \\
\hline Sample size & 279 & 117 \\
\hline \multicolumn{3}{|l|}{ Year of injury } \\
\hline Median & 1980 & 1981 \\
\hline Interquartile range & 1977 to 1984 & 1978 to 1985 \\
\hline \multicolumn{3}{|l|}{ Age at injury (years) } \\
\hline Mean & 24.9 & 21.5 \\
\hline SD & 15.6 & 14.2 \\
\hline Male & $210(75.3)$ & $81(69.2)$ \\
\hline \multicolumn{3}{|l|}{ Cause of injury } \\
\hline Road traffic accident & $181(65.8)$ & $80(68.4)$ \\
\hline Fall under influence of alcohol & $20(7.3)$ & $8(6.8)$ \\
\hline Other & $74(26.9)$ & $29(24.8)$ \\
\hline Major extracranial complication & $101(36.3)$ & $54(46.2)$ \\
\hline Lucid interval & $48(17.8)$ & $21(18.6)$ \\
\hline \multicolumn{3}{|l|}{ GCS at A\&E } \\
\hline $3-8$ & $184(82.9)$ & $88(89.8)$ \\
\hline $9-12$ & $17(7.7)$ & $4(4.1)$ \\
\hline $13-15$ & $21(9.5)$ & $6(6.1)$ \\
\hline Non-reacting pupils at A\&E & $24(10.4)$ & $15(15.3)$ \\
\hline \multicolumn{3}{|l|}{ Injury type* } \\
\hline Diffuse & $166(59.5)$ & $67(57.3)$ \\
\hline Evacuated mass & $97(34.8)$ & $43(36.8)$ \\
\hline Non-evacuated mass & $16(5.7)$ & $7(6.0)$ \\
\hline \multicolumn{3}{|l|}{ GOS at six months } \\
\hline Vegetative/severe disability & $73(26.2)$ & $28(24.1)$ \\
\hline Moderate disability & $86(30.8)$ & $40(34.5)$ \\
\hline Good recovery & $120(43.0)$ & $48(41.4)$ \\
\hline \multicolumn{3}{|l|}{ NART error score } \\
\hline $1-18$ & $62(22.2)$ & $24(20.5)$ \\
\hline $19-28$ & $69(24.7)$ & $27(23.1)$ \\
\hline $29-48$ & 65 (23.3) & $26(22.2)$ \\
\hline Not completed & $83(29.7)$ & $40(34.2)$ \\
\hline
\end{tabular}

Early clinical data for the 396 of 1133 patients who were successfully followed up for late assessment 15 to 25 years after injury, divided into APOE $\epsilon 4$ carriers and non-carriers. Values are $n$ or $n(\%)$.

* Pattern of injury from imaging and operative findings.

$A \& E$, accident and emergency department; GCS, Glasgow coma score; GOS, Glasgow outcome scale; NART, national adult reading test.

\section{Cognitive state}

Premorbid intellectual function was estimated with the national adult reading test (NART). Current orientation and function were assessed by the mini-mental state examination (MMSE) and neuropsychological tests sensitive to cognitive decline associated with head injury and the prodromal stage of dementia. Memory function was assessed with subtests of the Wechsler memory scale (WMS: logical memory, verbal and visual paired associates, and visual reproduction) and the graded naming test. Executive and attentional processes were assessed with the Stroop neuropsychological screening test, verbal fluency (letter and category generation), and the digit span test from the WMS.

\section{Emotional state}

Self reported depression, distress, and psychiatric symptoms were assessed respectively with the Beck depression inventory, general health questionnaire (GHQ-28), and symptom check list-90 (SCL-90).

Physical health, social, family and employment status A comprehensive questionnaire obtained data on a wide range of social and health variables including alcohol and drug use, subsequent head injuries, or stroke, and was completed by the patient, relative, or carer as appropriate.

\section{Outcome}

The extended Glasgow outcome scale (GOSE) ${ }^{24} 25$ was used to determine the level of disability and "overall" outcome.
Table 3 General assessment of late outcome according to APOE genotype

\begin{tabular}{lll}
\hline & \multicolumn{2}{l}{ APOE genotype } \\
\cline { 2 - 3 } & $\epsilon 4$ negative & $\epsilon 4$ positive \\
\hline MMSE, raw scores & $64(22.9)$ & $27(23.1)$ \\
$0-23$ & $74(26.5)$ & $30(25.6)$ \\
$24-27$ & $75(26.9)$ & $29(24.8)$ \\
$28-29$ & $66(23.7)$ & $31(26.5)$ \\
30 & $77(27.6)$ & $35(29.9)$ \\
MMSE, centile scores & $52(18.6)$ & $23(19.7)$ \\
$\leqslant 1 \%$ & $79(28.3)$ & $28(23.9)$ \\
$2-30 \%$ & $71(25.4)$ & $31(26.5)$ \\
$31-69 \%$ & $100(35.5)$ & $35(29.9)$ \\
$70-100 \%$ & $51(18.3)$ & $35(29.9)$ \\
Composite performance, centile scores & $66(23.7)$ & $21(17.9)$ \\
Test incomplete & $62(22.2)$ & $26(22.2)$ \\
$1-17 \%$ & $52(17.8)$ & $24(19.0)$ \\
$18-30 \%$ & $40(13.7)$ & $17(13.5)$ \\
$31-100 \%$ & $83(28.4)$ & $38(30.2)$ \\
GOSE at follow up* & $28(9.6)$ & $19(15.1)$ \\
Vegetative/lower severe disability & $41(14.0)$ & $15(11.9)$ \\
Upper severe disability & $48(16.4)$ & $13(10.3)$ \\
Lower moderate disability & & \\
Upper moderate disability & & \\
Lower good recovery & & \\
Upper good recovery & &
\end{tabular}

General assessment of functional outcome 15 to 25 years after a head injury for carriers and non-carriers of APOE $\in 4$. Values are $n(\%)$.

*These results are based on 418 patients: the 396 who underwent neuropsychological assessment together with a further 22 where GOSE and APOE genotype were available.

GOSE, extended Glasgow outcome scale; MMSE, mini-mental state examination.

\section{Statistical methods}

The scores for the individual neuropsychological tests were converted to centiles from available normative data. The tests were grouped into five domains (verbal memory, visual memory, attention, language, and executive function) and the centile scores averaged within each domain to give an overall score per domain. These five summary scores were in turn averaged to produce a single "composite performance score" after the method of Fox and colleagues. ${ }^{26}$ The method has the advantage of minimising type 1 error by reducing the number of statistical tests that would otherwise be conducted on the individual cognitive tests.

Analysis of the neuropsychological scores was hampered by the fact that some of the patients were unable to complete all the tests. To overcome this problem, the composite performance score and MMSE scores (both raw scores and centile norms) were grouped into approximate quartiles, assigning individuals who were unable to complete a test to the lowest quartile for that test. The formal analyses are based on these quartile scores.

The association between the possession of an APOE $\epsilon 4$ allele and cognitive function was assessed formally using ordinal logistic regression to adjust for the early clinical data. The response variables for these analyses were the quartile scores for MMSE (both raw scores and centile scores) and for the composite performance score. A similar analysis was used for the full GOSE score, with the one patient who was assessed as vegetative being grouped into the lower severe disability category. The results both with and without adjustment for key baseline variables are summarised in terms of the common odds ratio together with the corresponding 95\% confidence intervals. An odds ratio of 1 would correspond to no effect, and values greater than 1 would indicate that possession of an APOE $\epsilon 4$ allele is associated with poor function. 
Table 4 Ordinal logistic regression analyses assessing the relation between possession of one or more APOE $\epsilon 4$ alleles and function as assessed at late follow up

\begin{tabular}{|c|c|c|c|c|c|c|}
\hline \multirow[b]{2}{*}{ Outcome measure } & \multicolumn{3}{|c|}{ Unadjusted analysis } & \multicolumn{3}{|c|}{ Adjusted analysis* } \\
\hline & $\begin{array}{l}\text { Common odds } \\
\text { ratiot }\end{array}$ & $95 \% \mathrm{Cl}$ & $\mathrm{p}$ Value & $\begin{array}{l}\text { Common odds } \\
\text { ratiof }\end{array}$ & $95 \% \mathrm{Cl}$ & $\mathrm{p}$ Value \\
\hline MMSE, raw score & 0.94 & 0.64 to 1.39 & 0.772 & 1.18 & 0.72 to 1.95 & 0.507 \\
\hline MMSE, centile score & 1.07 & 0.73 to 1.58 & 0.733 & 1.31 & 0.80 to 2.16 & 0.288 \\
\hline Composite performance score & 0.97 & 0.66 to 1.44 & 0.896 & 1.07 & 0.62 to 1.83 & 0.807 \\
\hline GOSE $\ddagger$ & 1.19 & 0.82 to 1.72 & 0.356 & 1.36 & 0.84 to 2.19 & 0.212 \\
\hline
\end{tabular}

Statistical analysis of the data presented in table 3 of the general assessment of functional outcome 15 to 25 years after a head injury for carriers and non-carriers of of APOE $\epsilon 4$.

*Adjusted for year of injury, age at injury, Glasgow coma score and pupil reaction at the accident and emergency department, type of injury, Glasgow outcome scale at six months, and national adult reading test.

$\dagger$ A common odds ratio of greater than 1 would indicate that possession of an APOE $\epsilon 4$ allele is associated with poor function.

$\ddagger$ These results are based on 418 patients: the 396 who underwent neuropsychological assessment together with a further 22 where GOSE and APOE genotype were available.

$\mathrm{Cl}$, confidence interval; GOSE, extended Glasgow outcome scale; MMSE, mini-mental state examination.

\section{RESULTS}

From the initial cohort of 1133 patients who were alive at six months following their head injury, 242 (21.4\%) were confirmed as being dead by the time of the long term follow up (mean follow up interval 18 years). In all, 396 (35\%) were traced and assessed using the battery of neuropsychological tests. The remaining 495 patients $(43.7 \%)$ were not fully assessed for the following reasons: no trace $(n=182)$; traced but no response $(\mathrm{n}=138)$; patient refused $(\mathrm{n}=76)$; patient not in Scotland, or remote $(n=83)$; general practitioner advised us not to contact the patient $(\mathrm{n}=16)$.

Table 1 gives a summary of the early clinical data for these three groups of patients. Overall the patients who had detailed neuropsychological assessments were very similar to the "remainder" group. However, the patients who had died between six months after their injury and the time of the study differed substantially from the other two groups in the following ways: they were older at the time of injury; they were more likely to have sustained their injury as a result of a fall while under the influence of alcohol; they were less likely to have had a road traffic accident; they were more likely to have had an intracranial mass lesion; they were less likely to have had diffuse brain injury; and at six months after their injury they were more likely to have been in the vegetative/ severe disability group and less likely to have had a good recovery. These features, shown in table 1 to be associated with mortality, are in accord with expectations.

Table 2 gives the same early clinical information, together with NART scores, for the patients who were fully assessed, divided into APOE $\in 4$ carriers and non-carriers. Of the 396 patients, $117(29.5 \%)$ were APOE $\epsilon 4$ carriers. The APOE $\epsilon 4$ negative and positive groups were broadly similar, although the APOE $\epsilon 4$ positive patients were younger at the time of injury (mean (SD) age: 21.5 (14.2) v 24.9 (15.6) years; 95\% confidence interval $(\mathrm{CI}), 0.2$ to 6.5 years; $\mathrm{p}=0.039$ ), more were pedestrians in a road traffic accident, and more suffered major extracranial complications. More APOE $\epsilon 4$ carriers had a low Glasgow coma score (3-8) than non-carriers $(89.8 \% v$ $82.9 \% ; 95 \% \mathrm{CI},-0.8 \%$ to $14.7 \%$; $\mathrm{p}=0.11)$. There was little difference in their status six months after their injury as assessed by the Glasgow outcome scale, with $41.4 \%$ of APOE $\epsilon 4$ carriers having a good recovery compared with $43.0 \%$ of non-carriers.

The late functional outcome measures for the APOE $\epsilon 4$ carriers and non-carriers are summarised in table 3: $22.2 \%$ of APOE $\epsilon 4$ carriers had a good recovery according to their GOSE, compared with $30.5 \%$ non-carriers (95\% CI, $-0.7 \%$ to $17.2 \%$; $\mathrm{p}=0.084)$. There was no trend for the difference between carriers and non-carriers of APOE $\epsilon 4$ to be more marked at older ages; in fact for the under 50s the observed difference in the proportion with good recovery is $10 \%$, which is greater than the difference observed overall. There were no clear and consistent differences between the two groups for MMSE or the global impairment score. The results of the formal statistical comparisons using ordinal logistic regression with and without adjustment for the key clinical variables including GOSE at six months are shown in table 4. There was no evidence of any association between possession of APOE $\epsilon 4$ and late functional outcome using these measures.

The results of the neuropsychological assessments of late outcome for APOE $\epsilon 4$ carriers and non-carriers are shown in table 5. Although there was a slight tendency for carriers to be less likely to have performed well in tests of visual memory, attention, and language, there were no significant differences between the two groups (table 6). Among patients who were severely disabled at six months, APOE $\epsilon 4$ carriers performed consistently worse than non-carriers in their late assessments, but this tendency was not present among patients who were moderately disabled or had good recovery at six months (data not shown).

Table 5 Detailed neuropsychological assessments of late outcome according to APOE genotype

\begin{tabular}{lll}
\hline & \multicolumn{2}{l}{ APOE genotype } \\
\cline { 2 - 3 } & $\epsilon 4$ negative & $\epsilon 4$ positive \\
\hline $\begin{array}{lll}\text { Verbal memory, centile scores } \\
\text { Test incomplete }\end{array}$ & $70(25.1)$ & $28(23.9)$ \\
$1-4 \%$ & $70(25.1)$ & $33(28.2)$ \\
$4-18 \%$ & $77(27.6)$ & $21(17.9)$ \\
$18-100 \%$ & $62(22.2)$ & $35(29.9)$ \\
Visual memory, centile scores & & \\
Test incomplete & $90(32.3)$ & $29(24.8)$ \\
$1-10 \%$ & $50(17.9)$ & $29(24.8)$ \\
$10-25 \%$ & $64(22.9)$ & $31(26.5)$ \\
$25-100 \%$ & $75(26.9)$ & $28(23.9)$ \\
Attention, centile scores & & \\
Test incomplete & $49(17.6)$ & $22(18.8)$ \\
$1-30 \%$ & $93(33.3)$ & $41(35.0)$ \\
$30-50 \%$ & $56(20.1)$ & $30(25.6)$ \\
$50-100 \%$ & $81(29.0)$ & $24(20.5)$ \\
Language, centile scores & & \\
Test incomplete & $54(19.4)$ & $21(17.9)$ \\
$1-2 \%$ & $84(30.1)$ & $41(35.0)$ \\
$2-21 \%$ & $63(22.6)$ & $32(27.4)$ \\
$21-100 \%$ & $78(28.0)$ & $23(19.7)$ \\
Executive function, centile scores & & \\
Test incomplete & $87(31.2)$ & $33(28.2)$ \\
$1-8 \%$ & $57(20.4)$ & $29(24.8)$ \\
$8-25 \%$ & $73(26.2)$ & $30(25.6)$ \\
$25-100 \%$ & $62(22.2)$ & $25(21.4)$ \\
\hline
\end{tabular}

Detailed neuropsychological assessments of outcome in 396 subjects 15 to 25 years after a head injury for carriers and non-carriers of APOE $\epsilon 4$.

Values are $n(\%)$ 
Table 6 Ordinal logistic regression analyses of the relation between possession of one or more APOE $\epsilon 4$ alleles and detailed neuropsychological assessments at late follow up

\begin{tabular}{|c|c|c|c|c|c|c|}
\hline \multirow[b]{2}{*}{ Outcome measure } & \multicolumn{3}{|c|}{ Unadjusted analysis } & \multicolumn{3}{|c|}{ Adjusted analysis* } \\
\hline & $\begin{array}{l}\text { Common odds } \\
\text { ratiof }\end{array}$ & $95 \% \mathrm{Cl}$ & $\mathrm{p}$ Value & $\begin{array}{l}\text { Common odds } \\
\text { ratiot }\end{array}$ & $95 \% \mathrm{Cl}$ & $\mathrm{p}$ Value \\
\hline Verbal memory & 0.90 & 0.61 to 1.32 & 0.574 & 0.83 & 0.50 to 1.38 & 0.468 \\
\hline Visual memory & 0.92 & 0.63 to 1.36 & 0.690 & 0.94 & 0.56 to 1.56 & 0.806 \\
\hline Attention & 1.22 & 0.83 to 1.81 & 0.307 & 1.54 & 0.94 to 2.53 & 0.089 \\
\hline Language & 1.18 & 0.80 to 1.74 & 0.396 & 1.34 & 0.80 to 2.24 & 0.265 \\
\hline Executive function & 0.98 & 0.67 to 1.45 & 0.938 & 1.23 & 0.74 to 2.06 & 0.421 \\
\hline
\end{tabular}

Statistical analysis of the data presented in table 5 of the detailed neuropsychological assessments of outcome in 396 subjects 15 to 25 years after a head injury for carriers and non-carriers of $\mathrm{APOE} \in 4$.

*Adjusted for year of injury, age at injury, Glasgow coma score, and pupil reaction at the accident and emergency department, injury type, Glasgow outcome scale at six months, and national adult reading test score.

$\dagger A$ common odds ratio of greater than 1 would indicate that possession of an APOE $\epsilon 4$ allele is associated with poor function.

$\mathrm{Cl}$, confidence interval.

\begin{tabular}{|c|c|c|}
\hline & $\in 4$ negative & $\epsilon 4$ positive \\
\hline Improved & $15.8 \%$ & $12.7 \%$ \\
\hline Unchanged & $54.5 \%$ & $56.3 \%$ \\
\hline Deteriorated & $29.8 \%$ & $31 \%$ \\
\hline
\end{tabular}

Relative to their status at six months, approximately twice as many patients had deteriorated (126 patients, $30.2 \%, 95 \%$ CI, $28.0 \%$ to $32.5 \%$ ) as had improved ( 60 patients, $14.4 \%, 95 \%$ CI, $12.7 \%$ to $16.2 \%$ ) at late follow up (table 7 ). APOE $\epsilon 4$ carriers did not differ significantly in this respect from noncarriers.

\section{DISCUSSION}

In this study we show that a high proportion of surviving patients who were assessed 15 to 25 years after a head injury were severely impaired. Furthermore, comparison of the Glasgow outcome scale scores indicated that a deterioration occurred between the six month assessment and the late assessment in almost one third of cases, whereas improvement over this period was relatively uncommon. Late deterioration in this study was only measured by a functional outcome (the Glasgow outcome scale), and such an outcome may reflect various factors-for example, lack of rehabilitation. This finding supports previous studies suggesting that late deterioration may occur after head injury. ${ }^{6-8}$ An important advantage of the present study is that detailed comparisons could be made relating to the nature and severity of the injury, the early outcome, and the outcome many years later.

About $22 \%$ of APOE $\epsilon 4$ carriers had a good late outcome, compared with $31 \%$ of non-carriers (95\% CI for the difference, $-0.7 \%$ to $17.2 \% ; \mathrm{p}=0.084$ ), despite the fact that APOE $\epsilon 4$ carriers and non-carriers were very similar at their six month assessments after the injury. In view of the evidence that head injury is a risk factor for Alzheimer's disease, neuropsychological tests were chosen to target functions that are likely to be affected in the prodromal stages of Alzheimer's disease. These detailed tests were not done 15 to 25 years ago at the time of the initial six month follow up assessment, and so no direct comparison of these functions at the two time points is possible. Nonetheless, the use of centile scores derived in comparison with normative values for the tests to describe the degree of cognitive impairment does give an absolute measure of current functioning and confirms the profound impairment suffered by a large proportion of such patients. APOE $\epsilon 4$ carriers who in their early assessments were severely disabled according to the Glasgow outcome scale did show a tendency to perform consistently worse than non-carriers in the late neuropsychological tests, but this finding was not replicated among patients with moderate disability or good recovery at six months.

Important differences between the present study and that of Teasdale et al in $1997^{1}$ prevent a direct comparison of the findings. In the 1997 report we included admissions of all severities, and our indicator for an adverse association with the possession of APOE $\epsilon 4$ included death as an outcome. In contrast, the present study included only subjects who had survived for six months after their injury and who in addition were alive and available for follow up 15 to 25 years later. Only 396 patients from the original database of 1133 patients (35\%) could be traced and fully assessed, leaving considerable scope for selective loss of either APOE $€ 4$ carriers or non-carriers. Furthermore, we have recently shown in a prospective study of cardiac surgery patients that those who drop out from follow up of cognitive function are the ones with the greatest cognitive impairment, ${ }^{27}$ resulting in an underestimation of the prevalence and degree of impairment. Although a prospective study would be required to test this hypothesis fully, there is, however, evidence that this selective loss did not occur. Of the subjects assessed in this study, 117 of the 396 (29.5\%) were carriers of APOE $\epsilon 4$ and this is close to the values for our previous prospective studies of consecutively admitted patients with head injuries: $32.2 \%$ (30 of $93^{1}$ ) and $32.5 \%$ (349 of 1075; unpublished observations), respectively.

The role of the APOE genotype has been explored in relation to relatively short term outcome in the response to forms of acute brain injury other than trauma. For example, APOE $\epsilon 4$ has been found to be associated with poor outcome following spontaneous intracerebral haemorrhage, cardiopulmonary resuscitation, cardiac bypass surgery, and possibly subarachnoid haemorrhage, but not apparently after ischaemic stroke. ${ }^{28}{ }^{29}$ The mechanisms underlying these associations are unclear at present but both human and animal neuropathological studies have highlighted the importance of apoliprotein $\mathrm{E}$ in the acute response to brain injury. ${ }^{28}$ After brain injury there are marked alterations in the cellular localisation of apoliprotein E, most notably with an increase in neuronal immunoreactivity. Studies of genetically modified mice have provided further evidence that apoliprotein E modulates the response to injury. ${ }^{28}{ }^{29}$ For example, apoliprotein E deficient mice have more severe lesions than wild type mice, and mice bearing the human $\epsilon 4$ allele have more severe lesions than those with $\epsilon 3$. Apolipoprotein E seems to have a direct neuroprotective role, as neuronal damage after entorhinal cortex lesioning and global ischaemia in APOE deficient mice is ameliorated by intraventricular infusion of apoliprotein E. Mechanisms involving apoliprotein E which are of potential relevance to the response to acute injury ${ }^{28} 29$ concern deposition of amyloid $\beta$ protein, the maintenance of cytoskeletal 
integrity, protection from oxidative stress, and modulation of the response to excitotoxicity. APOE $\epsilon 4$ predisposes to atherosclerosis, ischaemic heart disease, cerebral amyloid angiopathy, and altered blood coagulation, which could compromise cardiovascular integrity in patients with injuries resulting from trauma. Of potential relevance to longer term outcome, apoliprotein $\mathrm{E}$ is involved in the delivery of cholesterol to neurones required for neurite outgrowth and synaptogenesis, ${ }^{30}$ clearance of degeneration products, and microglial activation, and in the maintenance of the cholinergic system. ${ }^{28}{ }^{29}$

Despite the 15 to 25 year period of follow up in this study, the subjects assessed were still relatively young (mean (SD) age, 42.1 (15.0) years) in the context of age related cognitive decline and the onset of sporadic Alzheimer's disease. Thus the study of World War II veterans which showed an association of head injury with Alzheimer's disease involved an interval between injury and assessment of approximately 50 years in subjects now aged mostly in their 70 s. $^{8}$ Likewise, patients in epidemiological studies of Alzheimer's disease which identified head injury as a risk factor were also older-with, for example, the onset of dementia at age 69.9 (9.0) years in the report by Guo et al. ${ }^{15}$ Even in a study which identified onset of dementia at an earlier age in Alzheimer's disease patients with a history of traumatic brain injury, ${ }^{14}$ the median age of onset was 76 years for men and 80 years for women. In the study reported here only 60 of the 396 patients assessed were over the age of 60 . The relatively small number of old subjects available for inclusion in this study may be in part because the early selection was biased towards younger patients, and in part because of the high rate of mortality in older subjects, both in the acute and the follow up phase. It is possible that if this cohort of head injured patients was to be assessed again at a later time point, a clearer relation of APOE genotype to cognitive decline might be found.

\section{ACKNOWLEDGEMENTS}

We thank Janice Stewart and Kathryn Brown for determination of APOE genotypes. The study was funded by the Medical Research Council (G9601296N).

\section{Authors' affiliations}

K Millar, Division of Behavioural Medicine, Department of Psychological Medicine, University of Glasgow, Gartnavel Royal Hospital, Glasgow, UK

J A R Nicoll, Division of Clinical Neurosciences, University of Southampton, Southampton General Hospital, Southampton, UK S Thornhill, G M Teasdale, Department of Neurosurgery, University of Glasgow, Southern General Hospital, Glasgow, UK

G D Murray, Public Health Sciences, Department of Community Health Sciences, University of Edinburgh Medical School, Edinburgh, UK

Competing interests: JARN is a named applicant for patent US5747260 "Method of prognosing chronic neurodegenerative pathology following a head injury" (May 1998).

\section{REFERENCES}

1 Teasdale GM, Nicoll JAR, Murray G, et al. Association of apolipoprotein E polymorphism with outcome after head injury. Lancet 1997:350:1069-71.

2 Sorbi S, Nacmias N, Piacentini S, et al. ApoE as a prognostic factor for post-traumatic coma. Nat Med 1995;1:852.
3 Friedman G, Froom P, Sazbon L, et al. Apolipoprotein E-epsilon4 genotype predicts a poor outcome in survivors of traumatic brain injury. Neurology 1999;52:244-8

4 Lichtman SW, Seliger G, Tycko B, et al. Apolipoprotein E and functional recovery from brain injury following postacute rehabilitation. Neurology 2000;55:1536-9.

5 Nicoll JAR, Roberts GW, Graham DI. Apolipoprotein E $\epsilon 4$ allele is associated with deposition of $\beta$-amyloid protein following head injury. Nat Med 1995;1:135-7.

6 Walker AE, Blumer D. The fate of World War II veterans with posttraumatic seizures. Arch Neurol 1989;46:23-6.

7 Corkin S, Rosen TJ, Sullivan EV, et al. Penetrating head injury in young adulthood exacerbates cognitive decline in later years. J Neurosci $1989 ; 9: 3876-83$

8 Plassman BL, Havlik RJ, Steffens DC, et al. Documented head injury in early adulthood and risk of Alzheimer's disease and other dementias. Neurology 2000;55:1158-66.

9 Newcombe F. Very late outcome after focal wartime brain wounds. $J$ Clin Exp Neuropsychol 1996;18:1-23.

10 Mortimer JA, Van Duijn CM, Chandra V, et al. Head trauma as a risk factor for Alzheimer's disease: a collaborative re-analysis of case-control studies. EURODEM Risk Factors Research Group. Int J Epidemiol 1991;20(suppl 2):S28-35.

11 Van Duijn CM, Clayton DG, Chandra V, et al. Interaction between genetic and environmental risk factors for Alzheimer's disease: a reanalysis of case-control studies. EURODEM Risk Factors Research Group. Genet Epidemiol 1994;1 1:539-51.

12 Mayeux R, Ottman R, Tang MX, et al. Genetic susceptibility and head injury as risk factors for Alzheimer's disease among community-dwelling elderly persons and their first-degree relatives. Ann Neurol elderly persons and

13 O'Meara ES, Kukull WA, Sheppard L, et al. Head injury and risk of Alzheimer's disease by apolipoprotein E genotype. Am J Epidemiol 1997; 146:373-84

14 Nemetz PN, Leibson C, Naessens JM, et al. Traumatic brain injury and time to onset of Alzheimer's disease: a population-based study. Am J Epidemiol 1999;149:32-40.

15 Guo Z, Cupples LA, Kurz A, et al. Head injury and the risk of AD in the MIRAGE study. Neurology 2000;54:1316-23.

16 Mehta KM, Ott A, Kalmijn S, et al. Head trauma and risk of dementia and Alzheimer's disease: the Rotterdam Study. Neurology 1999:53:1959-62.

17 Saunders AM, Strittmatter WJ, Schmechel D, et al. Association of apolipoprotein $\mathrm{E}$ allele epsilon 4 with late-onset familial and sporadic Alzheimer's disease. Neurology 1993;43:1467-72.

18 Mayeux R, Ottman R, Maestre G, et al. Synergistic effects of traumatic head injury and apolipoprotein-epsilon 4 in patients with Alzheimer's disease. Neurology 1995;45:555-7.

19 Jordan BD, Relkin NR, Ravdin LD, et al. Apolipoprotein E epsilon4 associated with chronic traumatic brain injury in boxing. JAMA 1997; 278:136-40

20 Corsellis JA. Boxing and the brain. BM 1989;298:105-9.

21 Jennett B, Bond $M$. Assessment of outcome after severe brain damage. Lancet 1975;i:480-4

22 Wenham PR, Price WH, Blandell G. Apolipoprotein E genotyping by one-stage PCR. Lancet 1991;337:1 158-9.

23 Teasdale G, Jennett B. Assessment of coma and impaired consciousness. A practical scale. Lancet 1974;ii:81-4.

24 Teasdale GM, Pettigrew LE, Wilson JT, et al. Analyzing outcome of treatment of severe head injury: a review and update on advancing the use of the Glasgow outcome scale. J Neurotrauma 1998;15:587-97.

25 Wilson JT, Pettigrew LE, Teasdale GM. Structured interviews for the Glasgow outcome scale and the extended Glasgow outcome scale: guidelines for their use. Neurotrauma 1998;15:573-85.

26 Fox NC, Warrington EK, Seiffer AL, et al. Presymptomatic cognitive deficits in individuals at risk of familial Alzheimer's disease. A longitudinal prospective study. Brain 1998;121:1631-9.

27 Millar K, Asbury AJ, Murray GD. Pre-existing cognitive impairment as a factor influencing outcome after cardiac surgery. Br J Anaesth 2001:86:63-7.

28 Graham DI, Horsburgh K, Nicoll JAR, et al. Apolipoprotein E and the response of the brain to injury. Acta Neurochir 1999;73:89-92.

29 Horsburgh K, McCarron MO, White F, et al. The role of apolipoprotein $\mathrm{E}$ in Alzheimer's disease, acute brain injury and cerebrovascular disease: evidence of common mechanisms and utility of animal models. Neurobiol Aging 2000;21:245-55.

30 Mauch DH, Nagler K, Schumacher S, et al. CNS synaptogenesis promoted by glia-derived cholesterol. Science 2001;294:1354-7. 